\title{
To scan or not to scan - D-dimers and computed tomography pulmonary angiography in the era of COVID-19
}

\author{
Authors: Alexander A Tuck, ${ }^{A *}$ Harriet L White, ${ }^{A *}$ Badr A Abdalla, ${ }^{B}$ Gwendolen J Cartwright, ${ }^{C}$ Katherine R Figg, ${ }^{C}$ \\ Emily N Murphy, ${ }^{C}$ Benjamin C Pyrke, ${ }^{A}$ Mark A Reynolds, ${ }^{D}$ Rana M Taha ${ }^{A}$ and Hasan N Haboubi ${ }^{E}$
}

\begin{abstract}
The COVID-19 pandemic has had many ramifications on healthcare delivery and practice. As part of this, utilising biomarkers to risk stratify patients has become increasingly popular. During the COVID-19 pandemic the use of D-dimer has increased due to the evidence of COVID-19 induced thrombo-embolic disease. We evaluated the use of D-dimer on all hospital admissions during the peak of the pandemic and evaluated its sensitivity in diagnosing pulmonary embolic disease (PE). Patients without COVID-19 infection were as likely to have evidence of PE as their COVID-positive counterparts. However, the sensitivity of a D-dimer was higher in COVID-positive patients at a lower D-dimer level $(>1,500 \mu \mathrm{g} / \mathrm{L}$, sensitivity $81 \%$, specificity $70 \%)$ than in those without clinical, immunological or radiological evidence of COVID-19 infection (D-dimer $>2,000 \mu \mathrm{g} / \mathrm{L}$, sensitivity $80 \%$, specificity $76 \%$ ). These data suggest higher D-dimer thresholds should be considered for the exclusion of pulmonary emboli.
\end{abstract}

KEYWORDS: D-dimer, CTPA, pulmonary embolism, COVID-19, sensitivity

DOI: $10.7861 /$ clinmed.2020-0664

\section{Introduction}

In December 2019 a novel coronavirus (SARS-CoV-2) was identified in Wuhan, China, which has developed into a global pandemic with widespread repercussions. SARS-CoV-2 causes COVID-19, which manifests as viral pneumonia in some patients and can lead to overactivation of the body's immune system, resulting in pulmonary fibrosis and respiratory failure. The high mortality rate

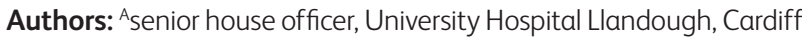
and Vale University Health Board; ${ }^{B}$ Clinical fellow gastroenterology, University Hospital Llandough, Cardiff and Vale University Health Board; Cfoundation year 1 doctor, University Hospital Llandough, Cardiff and Vale University Health Board; ${ }^{D}$ specialist registrar in gastroenterology, University Hospital Llandough, Cardiff and Vale University Health Board; ' ${ }^{E}$ consultant in gastroenterology, University Hospital Llandough, Cardiff and Vale University Health Board, and senior clinical lecturer, School of Medicine, Swansea University; *joint first authors of COVID-19 is attributed to a number of pathological processes, including diffuse alveolar damage, venous thromboembolism (VTE), superadded bacterial infection and heart failure.' Due to the rapid spread of COVID-19, the need for identification of prognostic factors was apparent and a number of laboratory tests have been suggested to predict mortality; one such test is D-dimer. ${ }^{2}$

Coagulopathy and venous thromboembolism have repeatedly been described as common complications of COVID-19.' Proposed aetiologies for this coagulopathy include endothelial dysfunction, increased pro-inflammatory cytokines ('cytokine storm') and severe hypoxaemia. As such, D-dimer testing has dramatically increased during the COVID-19 pandemic and is now an investigation used as part of the workup for suspected COVID-19 patients. This has meant that a large group of patients with a low clinical suspicion of pulmonary embolism (PE) have had D-dimers performed on admission. ${ }^{3}$ Consequently, we have observed a greater number of computed tomography pulmonary angiographies (CTPAs) being performed. This conveys an increased risk to patients from intravenous contrast and exposure to $\mathrm{X}$-ray radiation, ${ }^{4}$ and also impacts on resource management within the NHS.

D-dimer is a biomarker of the fibrinolytic system and interpreted as an indirect marker of thrombotic activity. In the process of thrombus generation, fibrinogen is cleaved by thrombin, and fibrin monomers then form polymers through the action of factor XIIIa crosslinking adjacent D domains. D-dimer molecules are subsequently released during the degradation of fibrin clots by plasmin. Therefore, the presence of intravascular D-dimer molecules is highly indicative of thrombus formation. ${ }^{5}$

D-dimer was first identified in the 1970s for evaluation of disseminated intravascular coagulation. Initially, laboratory tests were unable to distinguish between fibrinogen and products of fibrin degradation; however, the development of monoclonal antibody based assays allowed measurement of D-dimer alone. ${ }^{6}$ In more recent times, a number of methods have been used to measure D-dimer, including enzyme-linked immunofluorescent immunoassays (EIFAs), microplate enzyme-linked immunosorbent assays (ELISAs) and latex agglutination quantitated tests. ${ }^{\text {? }}$

The use of D-dimer in predicting venous thromboembolism has been controversial due to the difficulties in interpreting the result. Results were previously reported as 'positive' or 'negative', which was dependent on identifying a cut-off value. Initially a threshold of $500 \mu \mathrm{g} / \mathrm{L}$ was suggested, a level still used by most manufacturers and institutions. This is because numerous studies demonstrated a $100 \%$ sensitivity for VTE with this cut-off. 8,9 
However levels above $500 \mu \mathrm{g} / \mathrm{L}$ are highly non-specific, ${ }^{10}$ with many conditions causing abnormal results including malignancy, haemorrhage, trauma, sepsis ${ }^{11}$ and, more recently, COVID-19.12 D-dimer has become a test used to exclude VTE, rather than confirm it. The development of risk stratification tools such as the Wells' score meant that D-dimer was recommended only in low risk patients, to avoid unnecessary investigation for VTE. Age-adjusted values for $\mathrm{D}$-dimer have also been proposed, with varying evidence for $^{13}$ and against ${ }^{14}$ this.

More recently, laboratories have moved away from reporting D-dimer as positive or negative and have begun providing a quantitative value. This has resulted in further confusion for clinicians as to the correct way to interpret D-dimer, especially given its increased use on admission for all possible COVID-19 patients. We developed the hypothesis that we are overusing CTPA and that a higher D-dimer cut-off may prove more useful when attempting to risk-stratify for VTE. As a result of this, we aimed to establish a more useful way of interpreting D-dimer in the new COVID-19 era.

\section{Methods}

This was a retrospective, observational study looking at CTPA data across two major university hospitals during the COVID pandemic (University Hospital of Wales, and University Hospital Llandough), both within the Cardiff and Vale University Health Board catchment area, accounting for a population of almost 600,000 individuals.

Using our local radiology platform, SYNAPSE (FUJIFILM Medical Systems), we included all patients who had undergone a CTPA between 1 April 2020 and 6 June 2020. These dates were selected as being representative of the peak COVID-19 era in this region. ${ }^{15}$ All duplicate studies were excluded.

We used the online Welsh Clinical Portal (WCP) to obtain demographic data, biochemical and microbiological results, discharge summary letters and mortality information. Through SYNAPSE, we obtained chest X-ray and CTPA reports and collected data on PE diagnosis, COVID-19 diagnosis and reported COVID-19 severity. From WCP we cross-referenced results for COVID-19 polymerase chain reaction (PCR) swabs, D-dimer, C-reactive protein (CRP), procalcitonin and troponin-I from the initial bloods of the admission pertaining to the CTPA in question.

Assays used are shown in supplementary material S1. The study was registered on the Clinical Audit Database (9524).

\section{Data analysis}

Analyses were performed using the Statistical Package for the Social Sciences (SPSS v.23.0), IBM Corp, Amrok, New York, USA with significance set at $\mathrm{p}<0.05$.

In short, normality was tested using the one-sample KolmogorovSmirnov goodness of fit test. Non-normal continuous data were analysed using non-parametric tests (Mann Whitney $\mathrm{U}$ or Kruskal Wallis test). Group data were assessed using a Pearson $\chi^{2}$ test. Multivariable analysis was undertaken using a multivariable ANOVA to identify factors associated with a positive diagnosis of PE. Average values were reported as median (with 95\% confidence intervals).

\section{Results}

There were 578 CTPA requests during the search period, of which seven were duplicate requests, and 27 were subsequently
Table 1. Demographics and biochemical measured

variables of study participants

\begin{tabular}{|c|c|c|}
\hline \multirow[t]{2}{*}{ Gender } & Male & $260 / 544(47.8)$ \\
\hline & Female & $284 / 544(52.2)$ \\
\hline \multirow[t]{3}{*}{ Age } & Total & $60.30(95 \%$ CI 58.33-61.25) \\
\hline & Males & $61.45(95 \%$ CI 58.14-62.24) \\
\hline & Females & $58.40(95 \%$ CI 57.34-61.49) \\
\hline \multirow[t]{4}{*}{$\begin{array}{l}\text { COVID-19 } \\
\text { status }\end{array}$} & $\begin{array}{l}\text { Total diagnosed } \\
\text { with COVID-19 }\end{array}$ & $198 / 544(36.4)$ \\
\hline & $\begin{array}{l}\text { Suspected } \\
\text { COVID-19 }\end{array}$ & $261 / 544(48.0)$ \\
\hline & Positive swab & $153 / 544(28.1)$ \\
\hline & $\begin{array}{l}\text { Not swabbed/ } \\
\text { swab unavailable }\end{array}$ & $116 / 544(21.3 \%)$ \\
\hline \multirow{4}{*}{$\begin{array}{l}\text { Biochemical } \\
\text { data, } \\
\text { median } \\
(95 \% \mathrm{CI})\end{array}$} & D-dimer & $1,177 \mu \mathrm{g} / \mathrm{L}(3,498.8-2,598.7)$ \\
\hline & CRP & $23.5 \mathrm{pg} / \mathrm{ml}(53.11-67.4)$ \\
\hline & Procalcitonin & $0.06 \mu \mathrm{g} / \mathrm{L}(1.89-0.45)$ \\
\hline & Troponin I & $4 \mu \mathrm{g} / \mathrm{L}(3.55-295.05)$ \\
\hline
\end{tabular}

cancelled. This yielded a total study group of 544 patients. Total number of CTPA requests were noted to be higher than during the same time period between 2016-2019 (supplementary material S2), with a $50 \%$ increase in CTPA requests $(n=578)$ seen in 2020 compared to the previous year ( $n=386$ in 2019).

\section{Demographics}

The demographics of our participants are included in Table 1. There was equal gender distribution and there was no significant difference in age between genders $(p=0.319)$. As expected, the majority of patients $(55.9 \%)$ with a clinical suspicion of COVID had a positive swab $(p<0.001)$

\section{Biochemical evaluation}

As shown in Table 1, the median D-dimer value was 1,177 $\mu \mathrm{g} / \mathrm{L}$ ((95\% CI 2,598.7-3,498.8) and D-dimer was higher in patients with increasing age $(p<0.001)$.

Males had a higher D-dimer than females, with a median D-dimer of $1,331 \mu \mathrm{g} / \mathrm{L}(95 \%$ CI 2,994.74-4,998.63) compared to 1,081 $\mathrm{gg} / \mathrm{L}$ (95\% CI 1,904.54-2,932.39), $\mathrm{p}=0.009$ (Mann Whitney $\mathrm{U}$ test). However, although the level was noted to be higher in men compared to women, the proportion of abnormally high D-dimers was not higher in males compared to females ( $p=0.757$, Chi square). There was also no noted difference in COVID-19 status or age between the genders.

\section{Risk factors for pulmonary embolus}

PE was diagnosed in $15.8 \%$ of patients. A diagnosis of PE did not correlate with an increased risk of mortality $(p=0.854)$. Furthermore, COVID-19 severity as commented on in CTPA reporting did not increase risk of PE $(p=0.714)$. Those that 
Table 2. Risk factors for pulmonary embolism: univariate and multivariate analysis

\begin{tabular}{|c|c|c|c|c|}
\hline & & PE diagnosed $(n=86)(\%)$ & Univariate analysis & Multivariate analysis \\
\hline Total & & $86 / 544(15.8 \%)$ & & \\
\hline Gender (male) & & $49 / 86(57 \%)$ & 0.055 & \\
\hline Median age ( $95 \%$ confidence interval) & & $60.05(47.49-63.31)$ & & \\
\hline D-dimer $(n=65)$ & $<500 \mu \mathrm{g} / \mathrm{L}$ & $1 / 86(1.2 \%)$ & 0.008 & 0.05 \\
\hline & $\geq 500 \mu \mathrm{g} / \mathrm{L}$ & $64 / 86(74.4 \%)$ & & \\
\hline Adjusted D-dimer & Normal & $12 / 86(18.5 \%)$ & 0.187 & \\
\hline$(n=65)$ & Raised & $53 / 86(81.5 \%)$ & & \\
\hline CRP & $<5 \mu \mathrm{g} / \mathrm{ml}$ & $7 / 86(8.2 \%)$ & $<0.001$ & \\
\hline ( $\mathrm{n}=78$ measured) & $\geq 5 \mu \mathrm{g} / \mathrm{ml}$ & $71 / 86(82.6 \%)$ & & \\
\hline Troponin & Normal & $34 / 86(39.5 \%)$ & $<0.001$ & \\
\hline ( $n=59$ measured) & Elevated* & $25 / 86(29.1 \%)$ & & \\
\hline Procalcitonin & $<0.05$ & $13 / 86(15.1 \%)$ & 0.049 & 0.001 \\
\hline ( $n=48$ measured) & $\geq 0.05$ & $35 / 86(40.7 \%)$ & & \\
\hline & Mild & $3 / 86(3.5 \%)$ & & \\
\hline Radiological & Moderate & $4 / 86(4.7 \%)$ & 0.546 & \\
\hline$(n=86)$ & Severe & $16 / 86(18.6 \%)$ & & \\
\hline & Not mentioned & $63 / 86(73.3 \%)$ & & \\
\hline & Total diagnosed & $31 / 86\left(36 . x^{\prime} 0\right)$ & & \\
\hline COVID-19 status & Positive swab & $25 / 86(34.2 \%)$ & 0.348 & \\
\hline ( $n=198$ total COVID-19 diagnosed) & Not swabbed & $13 / 86(15.1 \%)$ & & \\
\hline Death & Yes & $12 / 86(14.0 \%)$ & 0.854 & \\
\hline$(n=86)$ & No & $74 / 86(86.0 \%)$ & & \\
\hline
\end{tabular}

*Elevated as per local cut offs.

had a PE had a median D-dimer value of 4,664.50 $\mu \mathrm{g} / \mathrm{L}((95 \%$ CI $2,731.48-11,252.94)$ compared to $1,101.00 \mu \mathrm{g} / \mathrm{L}$ ( (95\% CI $1,734.41-4,033.62)$ in those that did not have a PE $(p=0.008$, Mann Whitney $U$ test). Age-adjusted D-dimers demonstrated no significant correlation with having a PE (Table 2).

Multivariate analysis revealed the independent risk factors associated with a positive CTPA diagnosis to be D-dimer and elevated procalcitonin.

Risk factor analysis of the non-COVID-19 cohort We have assessed variables that may account for increased D-dimer, including recent surgery and active malignancy. Within the high D-dimer ( $>1,500 \mu \mathrm{g} / \mathrm{L})$ non-COVID-19 cohort $(\mathrm{n}=109)$, 11 patients had a current diagnosis of cancer (nine solid organ and two haematological) and three had had a surgical operation in the preceding 6 months. Median D-dimer levels were not statistically higher in patients with active cancer than their non-cancer counterparts $-4,239 \mu \mathrm{g} / \mathrm{L}(95 \%$ CI 2,317.7-8,555.2) compared to $4,292.5 \mu \mathrm{g} / \mathrm{L}$ (95\% CI 5,640.4-8,241.0), $p=0.532$. Furthermore, we did not observe a significant increase in PE diagnosis in these patients with cancer $(p=0.297)$. Patients who had a recent surgical operation did not have statistically different $D$-dimer $(p=0.507)$ and did not have a higher risk of PE $(p=0.963)$ in this cohort.

\section{Sensitivity and specificity of D-dimer testing}

In those with age adjusted D-dimers, there was no acceptable value that maintained a reasonable sensitivity and specificity (area under the ROC curve 0.514) (supplementary material S3). We therefore used non-adjusted D-dimer values to assess the sensitivity and specificity of a range of D-dimer data. We conducted subgroup analyses of COVID-19 and non-COVID-19 groups to establish whether the D-dimer cut-off in these groups should be different. For all patients a D-dimer cut-off of $500 \mu \mathrm{g} / \mathrm{L}$ obtained a sensitivity of $98.5 \%$ and a specificity of $12.0 \%$. In non-COVID-19 patients a D-dimer cut-off of $1,500 \mu \mathrm{g} / \mathrm{L}$ obtained a sensitivity of $79.5 \%$ and a specificity of $68.2 \%$, whereas a cut-off of $2,000 \mu \mathrm{g} / \mathrm{L}$ obtained a sensitivity of $79.5 \%$ and a specificity of $75.8 \%$. In the COVID-19 cohort cut offs of 1,500 $\mu \mathrm{g} / \mathrm{L}$ and $2,000 \mu \mathrm{g} / \mathrm{L}$ obtained sensitivities and specificities of $81.0 \%$ to $70.0 \%$ and $71.4 \%$ to $75.6 \%$ respectively (supplementary material $\mathrm{S} 4$ ).

In all cases, the area under the curve suggested good test accuracy. In order to obtain a sensitivity of near $80 \%$ and a specificity of approximately $70 \%$, a cut-off of $1,500 \mu \mathrm{g} / \mathrm{L}$ was adequate. When sub-groups were analysed for COVID-19 status, a similar sensitivity and specificity can be maintained using a cut-off of $1,500 \mu \mathrm{g} / \mathrm{L}$ in the COVID-19 group. In the non-COVID-19 group, the cut off could be extended to $2,000 \mu \mathrm{g} / \mathrm{L}$ while still maintaining 


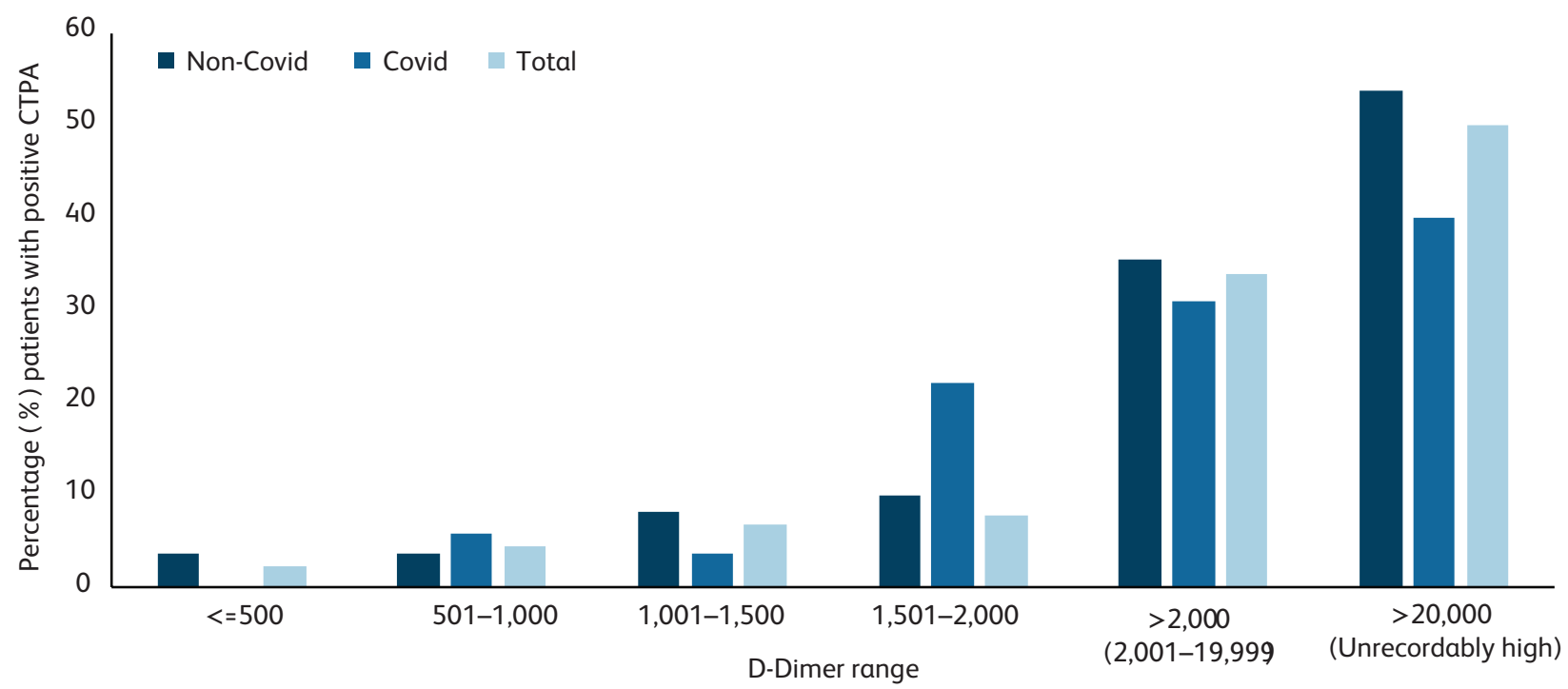

Fig 1. Percentage of patients with positive CTPA at different ranges of D-dimer.

sensitivity and improving specificity. Associated ROC curves for all three groups of data can be found in supplementary material S3.

Hence, a slightly lower D-dimer cut off should be considered in COVID-19 as there are greater numbers of PEs diagnosed in the 1,501-2,000 group in patients with COVID-19 compared to the non-COVID-19 cohort (Fig 1).

\section{Patient mortality}

Male gender was associated with an increased risk of death $(p=0.022)$, with a relative risk increase of 1.5 . Having a diagnosis of COVID-19 was also associated with an increased risk of death ( $p \leq 0.001$ ), with $67.1 \%$ of deaths having had a diagnosis of COVID-19. Increased age was associated with an increased risk of death with a median age of 69.10 years (95\% CI 66.52-71.76) in those that died versus 59 years (95\% CI 56.74-59.09) in those that survived, $p=0.022$ (Mann Whitney $U$ test). $D$-dimer greater than $500 \mu \mathrm{g} / \mathrm{L}(p=0.001)$, CRP greater than $5 \mathrm{pg} / \mathrm{ml}(p<0.001)$, elevated troponin $(p<0.001)$ and procalcitonin greater than $0.05 \mu \mathrm{g} / \mathrm{L}$ $(p<0.001)$ were associated with an increased risk of death.

COVID-19 severity on CTPA report was commented on in a total of 128 patients. Those with 'severe COVID-19' were more likely to die ( $p=0.003$, Chi Square). Having a raised D dimer increased the risk of death with a median D-dimer of $1,919 \mu \mathrm{g} / \mathrm{L}(95 \% \mathrm{CI}$ $2,079.55-7,190.54)$ in those that died compared to $1,020 \mu \mathrm{g} / \mathrm{L}$ (95\% CI 1,819.23-4,551.72) in those that survived, $p=0.005$ (Mann Whitney U-test).

\section{Discussion}

Our results show that the COVID-19 pandemic has coincided with a significant increase in the number of CTPAs compared to previous years. Although our yield of PE from CTPA (15.8\%) remained within a Royal College of Radiologists guideline of $15.4-37.4 \%{ }^{16}$ and was comparable with other radiology departments showing an average $14.6 \%{ }^{17}$ it was felt that better referral algorithms could be considered.
Our data show that the traditional D-dimer cut-off for further investigation for VTE of $500 \mu \mathrm{g} / \mathrm{L}$ resulted in a high sensitivity (98.5\%) but an unacceptably low specificity in all patients (12.0\%). This is similar to sensitivities and specificities of previous studies into D-dimer;, 8,9 however we support the argument that a higher uniform cut-off is now needed. Numerous studies have attempted to balance the differences in sensitivity and specificity ${ }^{10}$ that exist with D-dimer measurement. Recent studies have reported that lowering the target sensitivity can lead to a higher cut-off. As such, cut-offs of 900 and 1,200 have been suggested. ${ }^{18,19}$ A more recent 2019 latex agglutination-based study of 370 patients has reported that a significantly higher cut-off of $2,152 \mu \mathrm{g} / \mathrm{L}$ led to a sensitivity of $75.4 \%$ with a specificity of $62.7 \%$ for PE with a ROC AUC of $0.69(95 \%$ CI $0.64-0.74 ; \mathrm{p}<0.05) .{ }^{20}$ Our data suggest that a more moderate increased cut-off in all patients of 1,500 $\mu \mathrm{g} / \mathrm{L}$ could achieve similar sensitivities and specificities ( $80 \%$ and $69.2 \%$ respectively).

Our data also support different D-dimer cut offs for COVID-19 and non-COVID-19 patients. A D-dimer cut-off of 1,500 $\mu \mathrm{g} / \mathrm{L}$ in COVID-19 patients resulted in a sensitivity of $81 \%$ and specificity of $70 \%$, whereas $2,000 \mu \mathrm{g} / \mathrm{L}$ resulted in a similar sensitivity and specificity of $79.5 \%$ and $75.8 \%$ respectively in non-COVID-19 patients. COVID-19 specific data for D-dimer cut-offs is in its infancy, with many studies being based in ICU or with small numbers of patients. A recent study suggested a D-dimer cut-off of $>2,660 \mu \mathrm{g} / \mathrm{L}$ in COVID-19 patients (sensitivity $100 \%, 95 \%$ CI 88-100, and specificity of $67 \%, 95 \%$ CI 52-79) based on 106 COVID-19 patients that underwent CTPA examination..$^{21}$ Another study of 81 patients reported that a cut-off of $2,000 \mu \mathrm{g} / \mathrm{L}$ giving a sensitivity and specificity of $80 \%$ and $90.2 \%$ respectively.

We also suggest that a high D-dimer should elicit a high clinical suspicion of PE in non-COVID-19 and COVID-19 patients. In our data, D-dimer of $>2,000 \mu \mathrm{g} / \mathrm{L}$ had $33.9 \%$ risk of PE and greater than $20,000 \mu \mathrm{g} / \mathrm{L}$ resulted in a $50 \%$ risk of thromboembolism. This is in keeping with data showing that high D-dimer is an independent risk factor for PE. ${ }^{22}$

Furthermore, in our study the median D-dimer was $1,919 \mu \mathrm{g} / \mathrm{L}$ (95\% CI 2,079-7,190) in those that died compared to $1,020 \mu \mathrm{g} / \mathrm{L}$ 
(95\% CI 1,819-4,551) in those that survived. This supports current research that D-dimer is a useful prognostic marker for mortality, ${ }^{2}$ with previous studies reporting a D-dimer on admission $>2,000 \mu \mathrm{g} / \mathrm{L}$ was associated with a higher incidence of mortality $(P<0.001){ }^{23}$ This suggests that front-door $\mathrm{D}$-dimer testing is useful in the diagnostic work up of COVID-19 patients. However, our data also show that this has resulted in fewer $D$-dimers being requested with a Wells' Score. Immediately preceding COVID-19, D-dimer was recommended to be used in conjunction with a pre-test probability score such as the Wells or Geneva score. ${ }^{24}$ During the COVID-19 pandemic, routine 'front door' D-dimer testing was commenced due the measure's use as a prognostic marker. ${ }^{25}$ Therefore, most of our patients did not have pre-test probability of PE calculated in real time. Due to the retrospective nature of this study we could not subsequently calculate this due to missing data. Continued requesting of $\mathrm{D}$-dimer without pre-test probability is likely to lead to a significant increase in isolated elevated D-dimers in low-risk patients, which will remain a diagnostic challenge for clinicians in the absence of a higher cut-off.

We acknowledge the inherent limitations of this study. As this was a single-centre study, there is a possibility that local practice could influence the results. Its retrospective nature limited our ability to risk-stratify our patients due to lack of certain admission observations and examination information. Furthermore,

COVID-19 severity was based on CTPA reports, not on clinical severity. Although this is thought to correlate, ${ }^{26}$ it adds an element of subjectivity and inter-radiologist variation to the study. By selecting our study participants based on those who have had a CTPA, not those who have had a D-dimer, there will be patients with high D-dimers who did not have a CTPA; for example patients with severe renal impairment or those in whom it was not deemed to be in their best interests. This observation is further supported by our cohort of relatively young patients.

Additionally, the results of this study need to be interpreted in the context of an evolving understanding of a novel pathogen. It is understandable that clinicians may have been over-investigating due to the lack of clarity and understanding of complications of this disease.

Finally, due to different monoclonal antibody specificities, different D-dimer assay types are not directly comparable. ${ }^{27}$ Indeed, the significant under-reporting of D-dimer assays used in COVID-19 cohort data ${ }^{28}$ makes it difficult to relate utility of previous studies to a clinician's own institution. Therefore, in the use of our data clinicians need to be aware of how our institution's assay performance characteristics may be different from their own.

However, the study does have some notable strengths. It is one of the largest studies on this subject during the COVID-19 era and had very little missing data amongst the variables assessed. Furthermore, this study provides valuable 'front door' acute medicine data to help support clinician decision making.

\section{Conclusion}

Our research indicates how the COVID-19 era has changed clinical practice and suggests recommendations for improvements going forward. Our recommendation to increase the D-dimer cut-off is made in order to improve test specificity, understanding that a degree of sensitivity is therefore lost. This recommendation is rooted in the belief that $D$-dimer should form part of a thorough clinical assessment including a detailed history and examination, and high clinical suspicion - as defined in the modified Well's score - is enough of an indication for CTPA. This is particularly relevant in those with suspected or confirmed COVID-19 who are reported to be at greater risk of thromboembolic events.

We have demonstrated higher cut-offs of D-dimer can be used while maintaining acceptable levels of sensitivity and specificity, and that D-dimer thresholds may require adjustment depending on COVID-19 status. These findings support a more refined approach to D-dimer interpretation and the decision to scan or not. This could have positive implications through the reduction of iatrogenic complications of CTPA, more efficient resource allocation, and improved health economics.

Responding to a novel pathogen has required clinicians to adapt rapidly in order to treat those suffering with COVID-19. These adaptations have not just been directly in response to coronavirus, but also to changes in other pathways in the hospital. Our evidence indicates that not only do we have more to learn about coronavirus, but that we need to reflect on current clinical practice and find better and more efficient ways of working. It is our view that work conducted during the pandemic will inform lasting changes to the ways we practice medicine, and hope that innovations brought about by COVID-19 will long outlast the pandemic itself.

\section{Supplementary material}

Additional supplementary material may be found in the online version of this article at www.rcpjournals.org/clinmedicine:

S1 - Biochemical data assay details

S2 - Number of CTPA requests in the Cardiff and Vale University Health Board during the April to June period over the last 5 years S3 - ROC curves showing data all patients (age-adjusted), all patients (non-age-adjusted), COVID-19 patient and non-COVID-19 patient sub-groups

S4 - Sensitivity and specificity values for D-dimer cut offs.

\section{References}

1 Cui S, Chen S, Li X, Liu S, Wang F. Prevalence of venous thromboembolism in patients with severe novel coronavirus pneumonia. J Thromb Haemost 2020;18:1421-4.

2 Tang N, Li D, Wang X, Sun Z. Abnormal coagulation parameters are associated with poor prognosis in patients with novel coronavirus pneumonia. J Thromb Haemost 2020;18:844-7.

3 British Thoracic Society. BTS guidance on venous thromboembolic disease in patients with COVID-19. BTS, 2020.

4 Moore A, Wahsmann J, Chamarthy $M$ et al. Imaging of acute pulmonary embolism: an update. Cardiovasc Diagn Ther 2018;8:225243.

5 Weitz JI, Fredenburgh JC, Eikelboom JW. A test in context: D-dimer. J Am Coll Cardiol 2017;70:2411-20.

6 Greenberg C, Devine D, McCrae K. Measurement of Plasma Fibrin D-dimer levels with the use of monoclonal antibody coupled to latex beads. Am J Clin Pathol 1987;87:94-100.

7 Crawford F, Andras A, Welch K et al. D-dimer test for excluding the diagnosis of pulmonary embolism. Cochrane Database Syst Rev 2016;2016:CD010864.

8 Moerloose P, Desmarais S, Bounameaux $\mathrm{H}$ et al. Contribution of a new, rapid, individual and quantitative automated D-dimer ELISA to exclude pulmonary embolism. Thromb Haemost 1996;75:11-3.

9 Tardy B, Tardy-Poncet B, Viallon A et al. Evaluation of D-dimer ELISA test in elderly patients with suspected pulmonary embolism. Thromb Haemost 1998;79:38-41. 
10 Di Nisio M, Squizzato A, Rutjes A et al. Diagnostic accuracy of D-dimer test for exclusion of venous thromboembolism: a systematic review. J Thromb Haemost 2007;5:296-304.

11 Wells P. Integrated strategies for the diagnosis of venous thromboembolism. J Thromb Haemost 2007;5:41-50.

12 Yong L, Zhao K, Wei H et al. Dynamic relationship between D-dimer and COVID-19 severity. Br J Haematol 2020;190:e24-7.

13 Righini M, Van Es J, Den Exeter P et al. Age-adjusted D-dimer cutoff levels to rule out pulmonary embolism: the Adjust-PE study. JAMA 2014; 19;311:1117-24.

14 Lapner S, Julian J, Linkins L et al. Questioning the use of an age-adjusted D-dimer threshold to exclude venous thromboembolism: analysis of individual patient data from two diagnostic studies. J Thromb Haemost 2016:14:1953-9.

15 Public Health Wales. Rapid COVID-19 surveillance: confirmed case data. https://public.tableau.com/profile/public.health.wales. health.protection\#!/vizhome/RapidCOVID-19virology-Public/ Headlinesummary [Accessed 31 July 2020].

16 Quigley A, Brown K, Balasubramaniam R. Appropriateness of usage of computed tomography pulmonary angiography (CTPA) investigation of suspected pulmonary embolism. Royal College of Radiologists, 2017. www.rcr.ac.uk/audit/appropriateness-usage-computed-tomography-pulmonary-angiography-ctpa-investigation-suspected [Accessed 16 July 2020].

17 Mountain D, Keijzers G, Chu K et al. RESPECT-ED: rates of pulmonary emboli (PE) and sub-segmental pe with modern computed tomographic pulmonary angiograms in emergency departments: a multi-center observational study finds significant yield variation, uncorrelated with use of small PE rates. PLOS One 2016;11:e0166483.

18 Gupta R, Kakarla R, Kirshenbaum K, Tapson V. D-dimers and efficacy of clinical risk estimation algorithms: sensitivity in evaluation of acute pulmonary embolism. AJR Am J Roentgenol 2009;193:425-30.

19 Raviv B, Israelit S. Shifting up cutoff value of D-dimer in the evaluation of pulmonary embolism: a viable option? Possible risks and benefits. Emerg Med Int 2012;2012:517375.
20 Sikora-Skrabaka M, Skrabaka D, Ruggeri P et al. D-dimer value in the diagnosis of pulmonary embolism - may it exclude only? J Thorac Dis 2019;11:664-72.

21 Leonard-Lorant I, Delabranche X, Severac F et al. Acute pulmonary embolism in COVID-19 patients at CT angiography and relationship to D-dimer levels. Radiology 2020;296:E189-E191.

22 Schutte T, Thijs A, Smulders Y. Never ignore extremely elevated D-dimer levels: they are specific for serious illness. Neth J Med 2016;74:443-8.

23 Zhang L, Yan X, Fan Q et al. D-dimer levels on admission to predict in-hospital mortality in patients with COVID-19. J Thromb Haemost 2020;18:1324-9.

24 Hendriksen J, Geersing G, Lucassen W et al. Diagnostic prediction models for suspected pulmonary embolism: systematic review and independent external validation in primary care. $B M]$ 2015;351:h4438.

25 Tang N, Li D, Wang X, Sun Z. Abnormal coagulation parameters are associated with poor prognosis in patients with novel coronavirus pneumonia. J Thromb Haemost 2020;18:844-7.

26 Zhao W, Zhong Z, Xie X et al. Relation between chest CT findings and clincal conditions of coronoavirus disease (COVID-19) pneumonia: a multicenter study. Am J Roentgenol 2020;214:1072-7.

27 Goodwin AJ, Higgins RA, Moser KA et al. Issues surrounding age-adjusted D-dimer cutoffs that practicing physicians need to know when evaluating patients with suspected pulmonary embolism. Ann Intern Med 2017;166:361-4.

28 Favaloro E, Thachil J. Reporting of D-dimer data in COVID-19: some confusion and potential for misinformation. Clin Chem Lab Med 2020;28;58:1191-9.

Address for correspondence: Hasan Haboubi, Department of Gastroenterology, University Hospital Llandough, Penlan Rd, Llandough, Penarth CF64 2XX, UK.

Email: hasanhaboubi@doctors.org.uk 